\title{
Bacillus mycoides
}

National Cancer Institute

\section{Source}

National Cancer Institute. Bacillus mycoides. NCI Thesaurus. Code C86165.

A species of facultatively anaerobic, Gram-positive, rod shaped bacteria assigned to the phylum Firmicutes. This species is nonmotile, spore forming, catalase positive, oxidase negative, and hydrolyzes starch, gelatin, and oxidase. B. mycoides is found in soil. 